\title{
Absence of spontaneous magnetism associated with a possible time-reversal symmetry breaking state beneath the surface of (110)-oriented $\mathrm{YBa}_{2} \mathrm{Cu}_{3} \mathrm{O}_{7-\delta}$ superconducting films
}

\author{
H. Saadaoui, ${ }^{1}$ Z. Salman, ${ }^{1}$ T. Prokscha, ${ }^{1}$ A. Suter, ${ }^{1}$ H. Huhtinen, ${ }^{2}$ P. Paturi, ${ }^{2}$ and E. Morenzoni ${ }^{1}$ \\ ${ }^{1}$ Laboratory for Muon Spin Spectroscopy, Paul Scherrer Institut, 5232 Villigen PSI, Switzerland \\ ${ }^{2}$ Wihuri Physical Laboratory, Department of Physics and Astronomy, University of Turku, FI-20014 Turku, Finland
}

(Received 6 July 2012; revised manuscript received 17 October 2013; published 1 November 2013)

\begin{abstract}
We report the results of a search for spontaneous magnetism due to a time-reversal symmetry breaking phase in the superconducting state of (110)-oriented $\mathrm{YBa}_{2} \mathrm{Cu}_{3} \mathrm{O}_{7-\delta}$ films, expected near the surface in this geometry. Zero field and weak transverse field measurements performed using the low-energy muon spin rotation technique with muons implanted a few nm inside optimally doped $\mathrm{YBa}_{2} \mathrm{Cu}_{3} \mathrm{O}_{7-\delta}-(110)$ films show no appearance of spontaneous magnetic fields below the superconducting temperature down to $2.9 \mathrm{~K}$. Our results give an upper limit of $\sim 0.02 \mathrm{mT}$ for putative spontaneous internal fields.
\end{abstract}

DOI: 10.1103/PhysRevB.88.180501

PACS number(s): 74.25.Ha, 11.30.Qc, 73.22.Gk, 74.20.Rp

In unconventional superconductors, in addition to the global $U(1)$ phase rotation symmetry, other symmetries may be broken, leading to novel properties and multiple superconducting phases. ${ }^{1}$ A particularly interesting case is broken time-reversal symmetry (BTRS). A superconducting BTRS phase has been identified in few systems, such as $\mathrm{Sr}_{2} \mathrm{RuO}_{4},{ }^{2} \mathrm{PrOs}_{4} \mathrm{Sb}_{12},{ }^{3}$ $\mathrm{LaNiC}_{2},{ }^{4}$ and recently in $\mathrm{LaNiGa}_{2} .{ }^{5}$ A direct manifestation of BTRS is the appearance of spontaneous weak magnetic fields, ${ }^{6}$ detected in these systems by zero field muon spin relaxation $(\mathrm{ZF}-\mu \mathrm{SR}) .^{2-4}$ In high- $T_{c}$ superconductors, the nondegenerate spin-singlet $d_{x^{2}-y^{2}}$-wave ( $d$-wave) order parameter preserves time-reversal symmetry; thus ZF- $\mu$ SR and other techniques found no evidence of bulk BTRS. ${ }^{7-9}$

The bulk $d$-wave order parameter, however, can be suppressed near surfaces, defects, and boundaries, where a subcomponent order parameter can be stabilized leading in some cases to a BTRS state. ${ }^{6}$ Whereas the best conditions for the appearance of a detectable effect at defects or twin boundaries in $\mathrm{YBa}_{2} \mathrm{Cu}_{3} \mathrm{O}_{7-\delta}$ (YBCO) are not well clarified, ${ }^{10,11}$ BTRS is predicted to occur near a surface perpendicular to the 2-dimensional $\mathrm{CuO}_{2}$ planes, where the elastic scattering of the quasiparticles experiences a sign change of the pair potential along their trajectory in the $\mathrm{CuO}_{2}$ planes. ${ }^{12}$ This process [maximal in (110) orientation] leads to the formation of zeroenergy, current carrying surface Andreev bound states (ABS). These appear, in the quasiparticle density of states (DOS), as a zero bias conductance peak (ZBCP). ${ }^{13}$ The scattering leads to breaking of the $d$-wave Cooper pairs within a few coherence lengths from the interface, giving way to a subdominant pairing interaction of different symmetry (e.g., $s$ wave) and a BTRS complex order parameter such as $d+i s^{6,14}$ Emergence of a BTRS state at the surface is associated with a spontaneous magnetic field, and leads to a shift in the energies of the ABS in zero field, thus splitting the ZBCP.

The experimental evidence of BTRS beneath the surface of YBCO is still controversial. ZBCP splitting has been measured in some tunneling experiments at low temperatures $(\lesssim 10 \mathrm{~K}),{ }^{15-17}$ is absent in others indicating a predominant $d$-wave symmetry, ${ }^{18}$ or observed in unexpected orientations such as (100). ${ }^{19}$ Experiments carried out to detect directly the spontaneous magnetism have also led to contradicting results. Weak fields have been detected near the edges of thin $c$-axis
YBCO films [that mimics a (110) surface] below $T_{c}$ using SQUID magnetometry. ${ }^{20}$ Spontaneous magnetic flux was also measured near the asymmetric $45^{\circ}$ grain boundary of $c$-axis YBCO films in zero field. ${ }^{21}$ However, fractional flux quanta were observed by scanning SQUID microscopy near $c$-axis films but not in a BTRS-supporting (103) surface, ${ }^{22}$ while phase-sensitive measurements showed no evidence for a BTRS state. ${ }^{23}$ A $\beta$-NMR experiment measured the field distribution in a silver overlayer deposited on YBCO films and found weak linewidth broadening below the $T_{c}$ of YBCO ${ }^{24}$ However, the broadening is observed in different orientations, including $c$ axis, pointing to a different origin than BTRS.

These conflicting results call for a direct experiment to locally probe the magnetism associated with a possible BTRS state near a (110) surface. The $\mu \mathrm{SR}$ technique has been the most sensitive local probe to study BTRS appearing below $T_{c}$ under zero field conditions in all candidate superconductors. ${ }^{2-4}$ Weak spontaneous magnetic fields can be detected by implanting spin-polarized muons in the bulk and monitoring their spin depolarization which is very sensitive to the surrounding local field. A recently developed technique, low-energy muon spin rotation (LE- $\mu \mathrm{SR}$ ), extends this capability to thin films, near surface regions, and interfaces using muons of tunable $\mathrm{keV}$ energy, stopping at depths from a few $\mathrm{nm}$ to a few hundred $\mathrm{nm} .{ }^{25}$ In this Rapid Communication, we report ZF and weak transverse field (TF) measurements using LE- $\mu$ SR to directly measure the local magnetic field beneath the surface of YBCO-(110) films. We find no evidence of magnetism at depths from a few $\mathrm{nm}$ to $\sim 60 \mathrm{~nm}$ below the surface of YBCO, the region where the spontaneous magnetism is expected in a BTRS scenario. We estimate an upper limit of $0.02 \mathrm{mT}$ of the putative BTRS fields.

The measurements were performed at the $\mu E 4$ beamline of the Swiss Muon Source, ${ }^{26}$ at the Paul Scherrer Institute, in Switzerland. A high-energy beam of surface muons is moderated by solid Ar, emitting low-energy muons of few $\mathrm{eV}$. These muons are accelerated to $14 \mathrm{keV}$ and directed to the sample plate. The samples are mounted onto a plate which is electrically isolated from the cold finger of the cryostat, and biased to a high voltage ranging between -12.5 and $12.5 \mathrm{kV}$. This allows us to change the implantation energy of the muons between 1.5 to $26.5 \mathrm{keV}$. The LE- $\mu \mathrm{SR}$ measurements are 

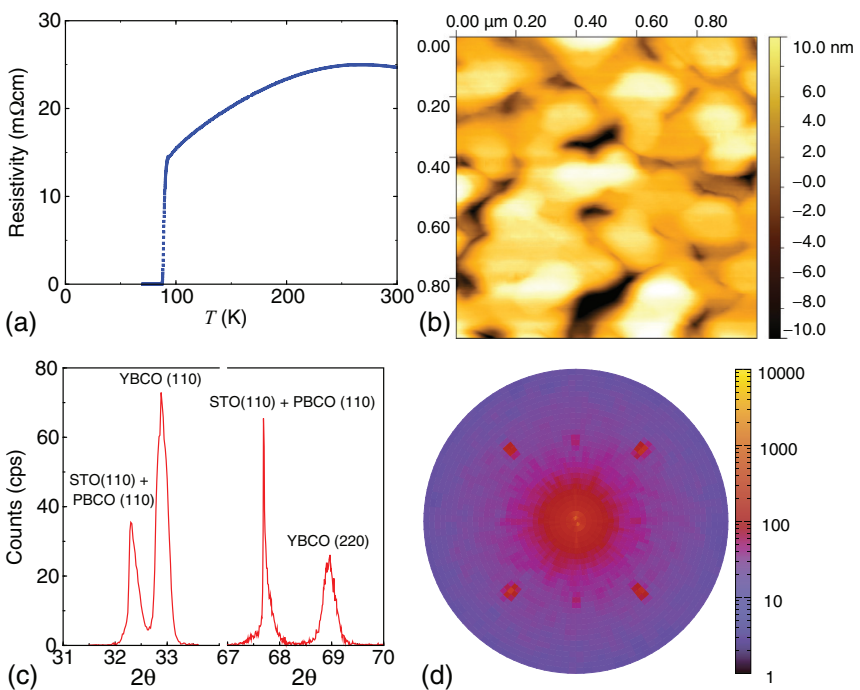

FIG. 1. (Color online) Typical characterization curves of the (110)-oriented films: (a) Resistivity along $c$ axis. (b) An AFM image of $1 \times 1 \mu \mathrm{m}^{2}$ area with an rms roughness of about $3 \mathrm{~nm}$. XRD Bragg peaks (c) and pole figure (d) confirming the (110) orientation of the films and absence of other phases.

performed in a temperature range from 2.9 to $150 \mathrm{~K}$. In $\mathrm{ZF}$, the stray magnetic fields at the sample position are less than $0.002 \mathrm{mT}$ in all directions. In weak TF, the field is applied perpendicular to both the initial muon spin polarization and beam direction, but parallel to the face of the sample. The time evolution of the polarization of the muon ensemble implanted in the sample, $P(t)$, measured via detection of the emitted decay positron intensity as a function of time after implantation, is very sensitive to the local magnetic environment.

The YBCO-(110) films were grown by pulsed laser deposition on (110) $\mathrm{SrTiO}_{3}$ (STO) single crystal substrates measuring $5 \times 5 \mathrm{~mm}$, with a $\mathrm{PrBa}_{2} \mathrm{Cu}_{3} \mathrm{O}_{7-x}(\mathrm{PBCO})$ template layer. The films are optimally doped with a transition temperature $T_{c}=$ 88.5 (1.0) K, as shown by resistivity measurements [Fig. 1(a)], whose temperature dependence is typical of $c$-axis resistivity. ${ }^{19}$ AFM shows a typical rms surface roughness of less than $3 \mathrm{~nm}$ over an area of a few square microns [Fig. 1(b)], but the films are atomically flat (rms roughness $\leqslant 1 \mathrm{~nm}$ ) on regions of order $0.1 \mu \mathrm{m}$ and covering about $80 \%$ of the surface. The overall rms roughness of $3 \mathrm{~nm}$ is due to small regions in between with vertical steps (nanocracks) of typical depth 10-20 nm. In this region the TRSB effect and associated magnetic field are expected to be smaller but not to disappear completely unless the orientation is (100), (010), or (001). ${ }^{14}$ Moreover, muons stopping in these nanocracks are still sensitive to the fields generated in the nearby flat parts of the surface. $\mathrm{X}$-ray diffraction (XRD) confirmed the crystallinity and single phase (110) of the films with less than $1 \%$ of other phases [Figs. 1(c), 1(d)]. Rutherford back scattering (RBS) was used to check the composition and thickness of the films and estimated the thickness of the YBCO films at $\sim 200 \mathrm{~nm}$. A mosaic of YBCO samples of a total area of $150 \mathrm{~mm}^{2}$ was mounted onto the sample holder with a good electric contact. Monte Carlo simulations using TRIM.SP ${ }^{27}$ show that the muon average implantation depth in YBCO ranges from 5 to $120 \mathrm{~nm}$

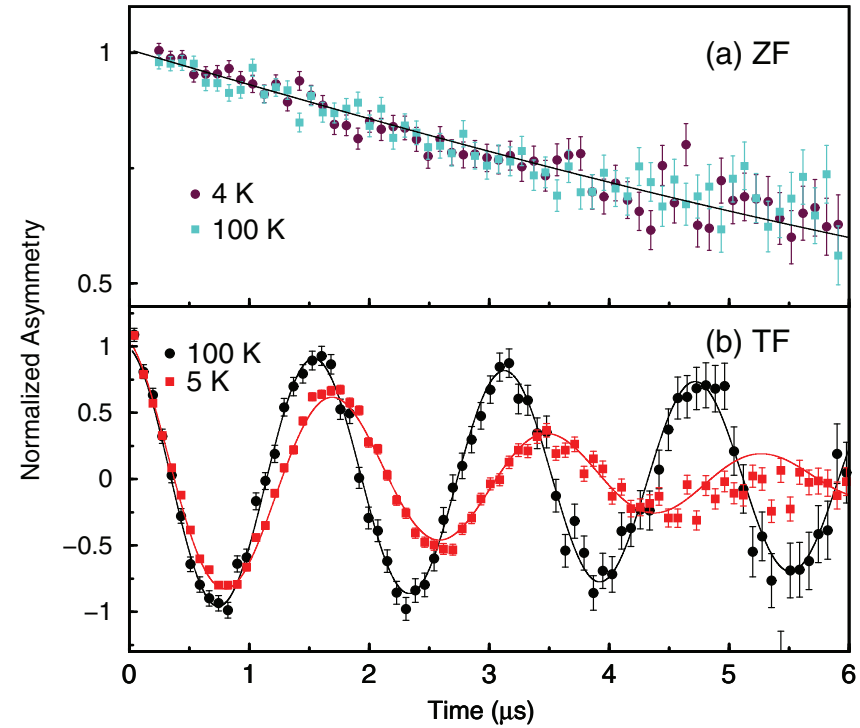

FIG. 2. (Color online) (a) Asymmetry in ZF at energy $2 \mathrm{keV}$, and temperature 100 and $4 \mathrm{~K}$. (b) Asymmetry in weak TF of $4.64 \mathrm{mT}$ (applied after ZF cooling) with muons of energy $14.2 \mathrm{keV}$ at 100 and $5 \mathrm{~K}$. Solid lines are fits explained in the text, and done using the MUSRFIT package (Ref. 28).

with a corresponding range straggling of 5 and $23 \mathrm{~nm}$, for implantation energies from 1 to $25 \mathrm{keV}$, respectively.

Typical ZF and TF $\mu$ SR spectra are presented in Fig. 2, above and below $T_{c}$. We start by discussing the TF data before focusing on the $\mathrm{ZF}$ results. The TF measurements, in a field of $4.64 \mathrm{mT}$ applied parallel to the surface, allow one to characterize the Meissner state by measuring the depth profile of the local field below the surface. The field is applied either parallel to the $c$ axis of YBCO, so that the shielding supercurrents flow in the $a b$ planes and one measures the in-plane London penetration depth $\lambda_{a b}$, or by rotating the sample by $90^{\circ}$ where the field is perpendicular to the $c$ axis with the shielding supercurrents flowing parallel to the $c$ axis, and one measures $\lambda_{c}$. The local field $B$ is obtained from the depth-dependent Larmor spin precession frequency $\omega=\gamma_{\mu} B$, where $\gamma_{\mu} / 2 \pi=135.5 \mathrm{MHz} / \mathrm{T}$ is the $\mu^{+}$gyromagnetic ratio. The samples in TF measurements are mounted on a nickel-plated sample holder, where the muons missing the sample depolarize very quickly (within few ns) and do not contribute to the polarization signal. ${ }^{29,30}$

Upon zero-field cooling below $T_{c}$ and then applying the TF, we see a significant reduction in the precession frequency as expected in the Meissner state. ${ }^{29}$ The mean internal field as a function of the beam energy at $5 \mathrm{~K}$ is plotted in Fig. 3 for an external field applied parallel to the $c$ axis. A fit using a cosine hyperbolic function, valid for thin films, ${ }^{31}$ yields $\lambda_{a b} \sim$ $160(10) \mathrm{nm}$. In a field perpendicular to the $c$ axis, we obtain $\lambda_{c} \sim 450(20) \mathrm{nm}$, thus confirming the overall (110) orientation of the films. The temperature dependence of the internal field is plotted in the inset of Fig. 3. The data are consistent with a single $d$-wave order parameter in the investigated region. ${ }^{32}$ From these weak-TF measurements, we can also rule out the appearance of strong spontaneous fields near the surface since in both orientations and throughout the film we observed only the diamagnetic shift characteristic of the Meissner state. 


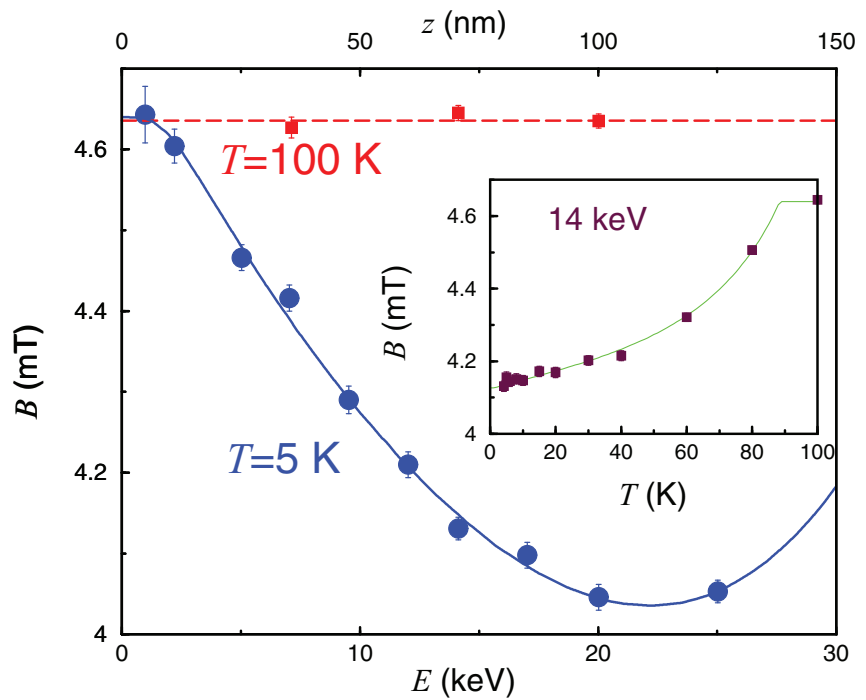

FIG. 3. (Color online) The energy dependence of the average magnetic field in the Meissner state at $5 \mathrm{~K}$. The applied field of $4.64 \mathrm{mT}$ as measured in the normal state at $100 \mathrm{~K}$ (dashed line) is applied parallel to the $c$-axis direction of YBCO films. The average depth corresponding to the muon energy as simulated by TRIM.SP is displayed on the top axis. Inset: Variation of the average magnetic field versus temperature with $14 \mathrm{keV}$ muons stopping at an average depth of $65 \mathrm{~nm}$. The solid line is a fit using a $d$-wave gap function (Ref. 32).

In bulk superconductors the most sensitive signature of spontaneous magnetism due to BTRS is a characteristic monotonic increase of $\mathrm{ZF}$ depolarization rates setting in below the critical temperature of the phase transition with BTRS. The ZF asymmetry in (110)-oriented YBCO films as presented in Fig. 2(a) shows no apparent difference in cooling below $T_{c}$. Beam energies of 2 and $4 \mathrm{keV}$ are chosen to maximize the number of probes stopping in the region where TRSB is expected. ${ }^{14}$ The asymmetry decays with a small depolarization rate ruling out the presence of large internal fields. If a long-range-ordered magnetic state is present, the $\mu \mathrm{SR}$ spectra should display precession at a frequency set by the average magnetic field at the muon site. In a disordered magnetic field state, the spin polarization decays faster with a rate set by the overall field width. We performed measurements of the ZF- $\mu$ SR asymmetry from 120 down to $2.9 \mathrm{~K}$, and fitted the data to the function ${ }^{2-4}$

$$
G(t)=A\left[\frac{1}{3}+\frac{2}{3}\left(1-\Delta^{2} t^{2}\right) e^{-\frac{1}{2} \Delta^{2} t^{2}}\right] e^{-\Lambda t}+A_{\mathrm{bg}} e^{-\lambda_{\mathrm{bg}} t},
$$

where the first term accounts for YBCO, and the second for the temperature-independent background signal from the muons missing the sample and hitting the Ag-coated sample holder used in the ZF experiment. ${ }^{30}$ The observed signal is dominated by YBCO (more than $50 \%$ fraction). Ag has a low relaxation rate $\left(\sim 0.02 \mu \mathrm{s}^{-1}\right)$, and its contribution to the signal has been measured separately and included in the fit function of Eq. (1). We have found similar spectra when the backing signal is nickel which relaxes completely the spins of the muons missing the sample. For the ZF case we preferred to present only the data on $\mathrm{Ag}$ as it is a nonmagnetic material itself. The depolarization in YBCO, caused by the nuclear moments (of width $\Delta=0.08 \mu \mathrm{s}^{-1}$ ), is accounted for by the Kubo-Toyabe function, multiplied by an exponential

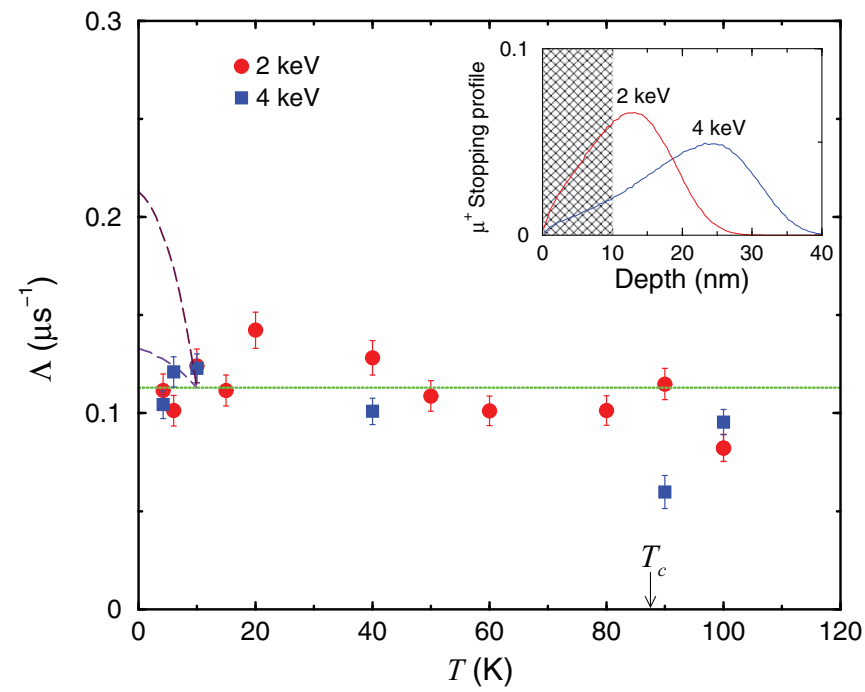

FIG. 4. (Color online) The electronic depolarization rate versus temperature with slow muons of energy 2 and $4 \mathrm{keV}$ implanted in YBCO. The dashed lines are guidelines representing the effect of BTRS fields of width 0.02 and $0.1 \mathrm{mT}$ on the ZF depolarization rate. Inset shows the stopping profile of the implanted muons, where the average rms depth for 2 and $4 \mathrm{keV}$ muons is 12(5) and $21(8) \mathrm{nm}$, respectively. Dashed region corresponds to a region where BTRS is expected.

accounting for the depolarization of electronic origin. ${ }^{2-4}$ The static depolarization rate, $\Lambda$, is proportional to the width of the local magnetic field distribution, $\Lambda \simeq \gamma_{\mu} \Delta B$.

The temperature variation of $\Lambda$ at 2 and $4 \mathrm{keV}$ is plotted in Fig. 4. This shows no noticeable increase of the depolarization rate below $T_{c}$ down to $2.9 \mathrm{~K}$. The results are consistent with a field distribution dominated by randomly oriented nuclear dipolar moments in YBCO. Experiments carried out on films with double the surface roughness (not shown here) yielded no difference with the measurements presented here, ruling out the effect of surface quality of the films on the depolarization rate in ZF. We can rule out the appearance of a BTRS state, as the spontaneous disordered magnetic fields would enhance $\Lambda$ upon entering the phase supporting BTRS as demonstrated by $\mu \mathrm{SR}$ in the bulk on other systems. ${ }^{2-4}$ For comparison, we plot in Fig. 4 the expected behavior of the depolarization rate for internal fields of $0.02 \mathrm{mT}$ and $0.1 \mathrm{mT}$ (dashed lines), which have been typically detected in BTRS states by ZF- $\mu$ SR. From the average scatter of the data one can draw an upper limit of $0.02 \mu \mathrm{s}^{-1}$ corresponding to spontaneous magnetic fields of $\sim 0.02 \mathrm{mT}$.

As mentioned above, the proposal of BTRS near (110) surfaces stems mainly from the prediction and observation of ZBCP splitting in zero field. ${ }^{14,15,19}$ Fogelström et al. showed in Ref. 14 that the scale of the field associated with the splitting is of the order of a fraction of the bulk critical field, $B_{0} \sim \phi_{0} / \pi^{2} \xi \lambda$. The depth of the region where spontaneous magnetism appears is not uniquely predicted. However, it is reasonable to assume an extent of a few coherence lengths in the $a b$ plane $\xi \simeq 2 \mathrm{~nm}$. At 2 and $4 \mathrm{keV}$, a sizable fraction of muons (30\% and 15\%, respectively) stop in this depth range (shadowed area, inset of Fig. 4). Moreover, also the muons 
stopping outside this region should still detect the stray fields. The width of the stray fields due to BTRS spontaneous fields extending over a length scale of $\xi$ may be approximated by $\Delta B \sim B_{\mathrm{s}}(\xi / z)^{3}$, where $B_{s}$ are estimates from tunneling, and $z$ is the muon stopping depth. Thus at an average depth of $12 \mathrm{~nm}$ at $2 \mathrm{keV}$, using an estimate of $B_{s}=0.1 B_{0} \approx 0.1 \mathrm{~T}$, we expect $\Delta B \sim 0.4 \mathrm{mT}$, much higher than our finding. Electron spin resonance performed with a $60 \mathrm{~nm}$ thick layer of active spins on YBCO (110) estimated the strength of stray fields at about $0.2 \mathrm{mT}^{33}$ A similar experiment performed by $\beta$-NMR with polarized ${ }^{8} \mathrm{Li}^{+}$spins implanted in an $\mathrm{Ag}$ overlayer on YBCO (110) drew an upper limit of BTRS at $0.02 \mathrm{mT}^{24}$ One has to notice that these experiments are done outside the superconductor; thus one expects a reduced sensitivity with respect to the present experiment.

The preferred muon site in $\mathrm{YBa}_{2} \mathrm{Cu}_{3} \mathrm{O}_{7-\delta}$ (close to the chain oxygen) and all possible secondary sites do not have lattice symmetry, so that a cancellation of fields from supercurrent with lattice symmetry can be excluded. ${ }^{34}$ Moreover, our experiment corresponds to the regime of zero transmission interface which significantly enhances BTRS and the corresponding ZBCP splitting ${ }^{35}$ and is free of complication of possible unusual proximity effects. ${ }^{36}$ These together with the fact that the measurements are performed directly in the YBCO make the present experiment particularly sensitive to magnetism. The absence of spontaneous BTRS fields in our experiment is in agreement with tunneling measurements where the $\mathrm{ZBCP}$ splitting was only observed in an applied field and vanishes in zero field. ${ }^{17,18,37-39}$

In conclusion, we have conducted a depth-resolved LE$\mu \mathrm{SR}$ study of the field distribution near the surface of YBCO films to look for direct evidence of spontaneous magnetism associated with a possible time-reversal symmetry complex order parameter that develops near a (110) surface. We detected no spontaneous internal fields in $\mathrm{YBCO}$, and consequently we established an upper limit of about $0.02 \mathrm{mT}$ for BTRS fields at low temperature. This field value is much weaker than that expected by tunneling experiments and various theories, where the ZBCP splitting is associated with much stronger spontaneous fields.

We thank M. Horisberger for depositing $\mathrm{Ni}$ and Ag on sample plates, M. Döbeli for performing RBS measurements, S. Rolf for assisting with AFM measurements, B. M. Wojek for helping with fitting, and R. F. Kiefl and M. Sigrist for useful discussion. The authors H.S. and E.M. acknowledge the financial support of the MANEP program, and H.H. and P.P., of the Wihuri Foundation.
${ }^{1}$ L. P. Gor'kov, Sov. Sci. Rev. A Phys. 9, 1 (1987); M. Sigrist and K. Ueda, Rev. Mod. Phys. 63, 239 (1991).

${ }^{2}$ G. M. Luke, Y. Fudamoto, K. M. Kojima, M. I. Larkin, J. Merrin, B. Nachumi, Y. J. Uemura, Y. Maeno, Z. Q. Mao, Y. Mori, H. Nakamura, and M. Sigrist, Nature (London) 394, 558 (1998).

${ }^{3}$ Y. Aoki, A. Tsuchiya, T. Kanayama, S. R. Saha, H. Sugawara, H. Sato W. Higemoto, A. Koda, K. Ohishi, K. Nishiyama, and R. Kadonom, Phys. Rev. Lett. 91, 067003 (2003).

${ }^{4}$ A. D. Hillier, J. Quintanilla, and R. Cywinski, Phys. Rev. Lett. 102, 117007 (2009).

${ }^{5}$ A. D. Hillier, J. Quintanilla, B. Mazidian1, J. F. Annett, and R. Cywinski, Phys. Rev. Lett. 109, 097001 (2012).

${ }^{6}$ M. Sigrist, Prog. Theor. Phys. 99, 899 (1998).

${ }^{7}$ R. F. Kiefl, J. H. Brewer, I. Affleck, J. F. Carolan, P. Dosanjh, W. N. Hardy, T. Hsu, R. Kadono, J. R. Kempton, S. R. Kreitzman, Q. Li, A. H. O'Reilly, T. M. Riseman, P. Schleger, P. C. E. Stamp, H. Zhou, L. P. Le, G. M. Luke, B. Sternlieb, Y. J. Uemura, H. R. Hart, and K. W. Lay, Phys. Rev. Lett. 64, 2082 (1990).

${ }^{8}$ G. J. MacDougall, A. A. Aczel, J. P. Carlo, T. Ito, J. Rodriguez, P. L. Russo, Y. J. Uemura, S. Wakimoto, and G. M. Luke, Phys. Rev. Lett. 101, 017001 (2008).

${ }^{9}$ J. E. Sonier, J. H. Brewer, R. F. Kiefl, R. H. Heffner, K. F. Poon, S. L. Stubbs, G. D. Morris, R. I. Miller, W. N. Hardy, R. Liang, D. A. Bonn, J. S. Gardner, C. E. Stronach, and N. J. Curro, Phys. Rev. B 66, 134501 (2002).

${ }^{10}$ M. Sigrist, D. B. Bailey, and R. B. Laughlin, Phys. Rev. Lett. 74, 3249 (1995).

${ }^{11}$ J.-X. Zhu and C. S. Ting, Phys. Rev. B 60, R3739 (1999).

${ }^{12}$ C. R. Hu, Phys. Rev. Lett. 72, 1526 (1994).

${ }^{13}$ For a review see G. Deutcher, Rev. Mod. Phys. 77, 109 (2005).
${ }^{14}$ M. Fogelström, D. Rainer, and J. A. Sauls, Phys. Rev. Lett. 79, 281 (1997).

${ }^{15}$ M. Covington, M. Aprili, E. Paraoanu, L. H. Greene, F. Xu, J. Zhu, and C. A. Mirkin, Phys. Rev. Lett. 79, 277 (1997).

${ }^{16}$ Y. Dagan and G. Deutscher, Phys. Rev. Lett. 87, 177004 (2001).

${ }^{17}$ G. Elhalel, R. Beck, G. Leibovitch, and G. Deutscher, Phys. Rev. Lett. 98, 137002 (2007).

${ }^{18}$ J. Y. T. Wei, N.-C. Yeh, D. F. Garrigus, and M. Strasik, Phys. Rev. Lett. 81, 2542 (1998).

${ }^{19}$ R. Krupke and G. Deutscher, Phys. Rev. Lett. 83, 4634 (1999).

${ }^{20}$ R. Carmi, E. Polturak, G. Koren, and A. Auerbach, Nature (London) 404, 853 (2000).

${ }^{21}$ J. Mannhart, H. Hilgenkamp, B. Mayer, Ch. Gerber, J. R. Kirtley, K. A. Moler, and M. Sigrist, Phys. Rev. Lett. 77, 2782 (1996).

${ }^{22}$ F. Tafuri and J. R. Kirtley, Phys. Rev. B 62, 13934 (2000).

${ }^{23}$ W. K. Neils and D. J. Van Harlingen, Phys. Rev. Lett. 88, 047001 (2002).

${ }^{24}$ H. Saadaoui, G. D. Morris, Z. Salman, Q. Song, K. H. Chow, M. D. Hossain, C. D. P. Levy, T. J. Parolin, M. R. Pearson, M. Smadella, D. Wang, L. H. Greene, P. J. Hentges, R. F. Kiefl, and W. A. MacFarlane, Phys. Rev. B 83, 054504 (2011).

${ }^{25}$ E. Morenzoni, H. Glückler, T. Prokscha, H. P. Weber, E. M. Forgan, T. J. Jackson, H. Luetkens, Ch. Niedermayer, M. Pleines, M. Birke, A. Hofer, J. Litterst, T. Riseman, and G. Schatz, Physica B 289, 653 (2000).

${ }^{26}$ T. Prokscha, E. Morenzoni, K. Deiters, F. Foroughi, G. George, R. Kobler, A. Suter, and V. Vrankovic, Nucl. Instrum. Methods Phys. Res., Sect. A 595, 317 (2008).

${ }^{27}$ W. Eckstein, Computer Simulation of Ion-Solid Interactions (Springer, Berlin, 1991). 
${ }^{28}$ A. Suter and B. M. Wojek, Phys. Proc. 30, 69 (2012).

${ }^{29}$ R. F. Kiefl, M. D. Hossain, B. M. Wojek, S. R. Dunsiger, G. D. Morris, T. Prokscha, Z. Salman, J. Baglo, D. A. Bonn, R. Liang, W. N. Hardy, A. Suter, and E. Morenzoni, Phys. Rev. B 81, 180502(R) (2010).

${ }^{30}$ H. Saadaoui, Z. Salman, T. Prokscha, A. Suter, B. M. Wojek, and E. Morenzoni, Phys. Proc. 30, 164 (2012).

${ }^{31}$ T. J. Jackson, T. M. Riseman, E. M. Forgan, H. Glückler, T. Prokscha, E. Morenzoni, M. Pleines, Ch. Niedermayer, G. Schatz, H. Luetkens, and J. Litterst, Phys. Rev. Lett. 84, 4958 (2000).

${ }^{32}$ R. Prozorov and R. W. Giannetta, Supercond. Sci. Technol. 19, R41 (2006).

${ }^{33}$ L. H. Greene, M. Aprili, M. Covington, E. Badica, D. E. Pugel, H. Aubin, Y.-M. Xia, M. B. Salamon, Sha Jain, and D. G. Hinks, J. Supercond. 13, 703 (2000).
${ }^{34}$ Ch. Niedermayer, H. Glückler, R. Simon, A. Golnik, M. Rauer, E. Recknagel, A. Weidinger, J. I. Budnick, W. Paulus, and R. Schöllhorn, Phys. Rev. B 40, 11386 (1989).

${ }^{35}$ Y. Tanuma, Y. Tanaka, and S. Kashiwaya, Phys. Rev. B 64, 214519 (2001).

${ }^{36}$ A. Kohen, G. Leibovitch, and G. Deutscher, Phys. Rev. Lett. 90, 207005 (2003)

${ }^{37}$ M. Aprili, E. Badica, and L. H. Greene, Phys. Rev. Lett. 83, 4630 (1999).

${ }^{38}$ R. Beck, Y. Dagan, A. Milner, A. Gerber, and G. Deutscher, Phys. Rev. B 69, 144506 (2004).

${ }^{39}$ N.-C. Yeh, C.-T. Chen, G. Hammerl, J. Mannhart, A. Schmehl, C. W. Schneider, R. R. Schulz, S. Tajima, K. Yoshida, D. Garrigus, and M. Strasik, Phys. Rev. Lett. 87, 087003 (2001). 